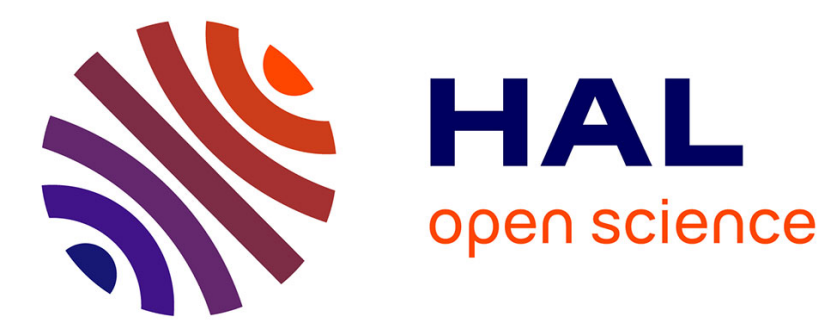

\title{
Coffee and tea intake and risk of breast cancer
}

Nirmala Bhoo Pathy, Petra H. M. Peeters, Carla H. van Gils, Joline W. J.

Beulens, Yolanda van Der Graaf, Bas Bueno-De-Mesquita, Awang Bulgiba, Cuno S. P. M. Uiterwaal

\section{- To cite this version:}

Nirmala Bhoo Pathy, Petra H. M. Peeters, Carla H. van Gils, Joline W. J. Beulens, Yolanda van Der Graaf, et al.. Coffee and tea intake and risk of breast cancer. Breast Cancer Research and Treatment, 2009, 121 (2), pp.461-467. 10.1007/s10549-009-0583-y . hal-00535404

\section{HAL Id: hal-00535404 https://hal.science/hal-00535404}

Submitted on 11 Nov 2010

HAL is a multi-disciplinary open access archive for the deposit and dissemination of scientific research documents, whether they are published or not. The documents may come from teaching and research institutions in France or abroad, or from public or private research centers.
L'archive ouverte pluridisciplinaire HAL, est destinée au dépôt et à la diffusion de documents scientifiques de niveau recherche, publiés ou non, émanant des établissements d'enseignement et de recherche français ou étrangers, des laboratoires publics ou privés. 


\title{
Coffee and tea intake and risk of breast cancer
}

\author{
Nirmala Bhoo Pathy - Petra Peeters - Carla van Gils • \\ Joline W. J. Beulens • Yolanda van der Graaf • Bas Bueno-de-Mesquita • \\ Awang Bulgiba $\cdot$ Cuno S. P. M. Uiterwaal
}

Received: 3 July 2009/Accepted: 7 October 2009/Published online: 22 October 2009

(C) Springer Science+Business Media, LLC. 2009

\begin{abstract}
Known risk factors account for about 10-15\% of breast cancer incidence suggesting that lifestyle exposures are crucial in its etiology. Previous epidemiological studies on the association between coffee and tea consumption and breast cancer risk have been inconsistent. We investigated the association of coffee and tea consumption with the risk of breast cancer among women in EPIC-NL cohort, a population-based prospective cohort in Netherlands with 27,323 participants. Exposure was measured by a validated food frequency questionnaire, and the outcome was verified by direct linkage with the Netherlands Cancer Registry. A total of 681 invasive primary breast cancers were diagnosed in 9.6 years of follow-up. Coffee intake increased the risk of breast cancer by more than twofold as compared to non-consumers (HR; 2.25, 95\% CI; 1.30 3.90). This association did not hold after multivariate adjustment which resulted in a HR of $1.17,95 \%$ CI; $0.65-$ 2.12. After adjustment to breast cancer risk factors and lifestyle, no association was observed between intake of coffee or tea and risk of breast cancer across all categories
\end{abstract}

N. Bhoo Pathy $\cdot$ P. Peeters · C. van Gils .

J. W. J. Beulens · Y. van der Graaf · C. S. P. M. Uiterwaal (ه) Julius Center for Health Sciences and Primary Care, University Medical Center Utrecht, Stratenum 6.131, P.O. Box 85500, 3508 GA Utrecht, The Netherlands

e-mail: C.S.P.M.Uiterwaal@umcutrecht.nl

N. Bhoo Pathy · A. Bulgiba

Department of Social and Preventive Medicine, Faculty of Medicine, University of Malaya, Kuala Lumpur, Malaysia

J. W. J. Beulens - B. Bueno-de-Mesquita

National Institute of Public Health and the Environment

(RIVM), Bilthoven, The Netherlands of intake. These results were also not altered by body mass index (BMI). Coffee and tea consumption does not seem to be related to the risk of breast cancer in women.

Keywords Coffee $\cdot$ Tea $\cdot$ Breast cancer

\section{Introduction}

Breast cancer is the most common type of female malignancy which results in significant morbidity and mortality across the globe. Currently, known risk factors in conjunction explain only some $10-15 \%$ of breast cancer incidence. Therefore, lifestyle and environmental exposures may be crucial in the etiology of breast cancer as suggested by international variation in breast cancer incidences and evidence from studies of migrants [1].

Coffee and tea are the world's most popular beverages, with The Netherlands being one of the top 10 countries in the world for coffee consumption per capita. In 2007, the World Cancer Research Fund (WCRF) concluded in its report that for the association between premenopausal and postmenopausal breast cancer with dietary exposures such as coffee and tea intake, the data were either of too low quality, or too inconsistent, or the number of studies were too few to allow conclusions to be reached [2]. While some case-control studies showed no association between coffee and tea intake and the risk of breast cancer [3-7], other studies have shown a protective effect [8-10] or a harmful effect [11]. Meanwhile, data from large prospective studies are limited with most of them reporting no association between coffee and/or tea and risk of breast cancer [12-18], except for a Norwegian study which showed a significant inverse association among lean women and a non-significant positive effect among the obese [19]. 
Association between coffee or tea consumption and the risk of breast cancer is biologically plausible since these beverages are a complex mixture of chemicals, e.g., caffeine and polyphenolic compounds such as flavonoids and lignans [20]. The hypothesis that caffeine may increase the risk of breast cancer was coined in 1970s and 1980s. Caffeine was found to be carcinogenic in animal models [21] possibly caused by an inhibitory effect of caffeine on the repair of UV-damaged DNA, and an enhancing effect on cytotoxic and mutagenic activities of alkylating agents [22]. On the contrary, flavonoids and lignans are members of a diverse group called phytoestrogens, which have similar structural properties with estradiol and may also act as estrogen antagonists [23]. Phytoestrogens have also been shown in vitro to exhibit a plethora of different anti-cancer effects, including proliferation of malignant cells through inhibition [24]. Lignans may also protect against breast cancer by modulation of local estradiol synthesis by inhibiting 17beta-HSD type 1 enzyme which is necessary for estrogen synthesis [25].

Therefore, we investigated the association of coffee and tea consumption with the risk of breast cancer within the EPIC-NL cohort which is a large population-based prospective cohort in The Netherlands, while enabling solid confounder adjustment.

\section{Methods}

Study population and assessment

The EPIC-NL study consists of the Prospect and MORGEN cohorts that cover the Dutch contribution to the European Prospective Investigation into Cancer and Nutrition (EPIC) cohort [26]. In brief, Prospect is a prospective cohort study of women, aged 49-70, who participated in the breast cancer screening between 1993 and 1997 [27]. The MORGEN cohort consists of men and women aged 20-59 years recruited from three Dutch cities (Amsterdam, Doetinchem, and Maastricht) [28] between 1993 and 1997. There are 40,011 participants in the EPICNL cohort.

At baseline, a general questionnaire containing questions on demographic characteristics and the risk factors and presence of chronic diseases were completed, together with a food frequency questionnaire. Although the general questionnaires from both cohorts were not identical, very similar information was reported. Coding of this information was standardized and merged into one uniform database.

The present analysis was restricted to only those women with no prior history of cancer, as well as those with complete information on coffee and tea intake. Initially,
29,751 women were available out of which 27,439 did not have a prior history of cancer, but 116 of them had to be excluded due to incomplete coffee or tea data, leaving 27,323 women for analysis. Those with prior history of cancer were excluded since they were likely to have changed their lifestyle habits, while the previous lifestyle habits may still have a large impact on subsequent second cancer risk.

\section{Exposure assessment}

Daily food intake was assessed using a validated food frequency questionnaire (FFQ) including questions on the usual frequency consumption of 77 main food items during the year preceding enrollment. Overall, the questionnaire allows the estimation of the average daily consumption of 178 food types. It was validated against twelve 24-h recalls before the start of the study among 121 men and women $[29,30]$. This FFQ was used to assess the daily amount of coffee and tea consumption during the preceding year to enrollment of the study. Participants were asked how many cups $(250 \mathrm{ml})$ of coffee and glasses of tea they consumed on the basis of average per day/per week/per month/per year. Data of the validation study by Ocke et al. [29] were used to estimate reliability of the coffee and tea consumption assessment. A Spearman correlation coefficient of 0.74 for coffee consumption and 0.87 for tea consumption was observed between the FFQ and the twelve 24-h dietary recalls. For the present analysis, the amount of coffee and tea consumption was divided into six categories, 0 cup, $0.1-1.0$ cups (reference group), 1.1-2.0 cups, 2.1-3.0 cups, 3.1-5.0 cups, and $>5.0$ cups per day. Since individuals who consumed coffee often also consumed tea and vice versa, we decided to combine coffee and tea intake for further analysis. The number of cups of coffee and tea reported by an individual was combined into seven categories i.e., group-0 (no tea and coffee), 1 (tea only), 2 (coffee only), 3 (moderate tea and moderate coffee intake), 4 (moderate tea and high coffee intake), 5 (high tea and moderate coffee intake), 6 (high tea and coffee intake) with moderate intake referring to 0.1-3.0 cups per day, while intake of $>3$ cups per day was considered as high.

Even though we had information on decaffeinated coffee, it was not separately analyzed since the numbers were too small to allow for a meaningful analysis.

Ascertainment of breast cancer cases and follow-up of the cohort

The outcome of interest in this study was the occurrence of first, primary invasive breast cancer. Data on diagnosis of breast cancer were obtained from the Netherlands Cancer Registry, which holds a standard computerized register of 
cancer patients. This database was directly linked to the EPIC-NL cohort with patients' names after obtaining their consents. Information on vital status and participant movements was made available through linkage with the municipal administration registries. The MORGEN cohort was censored on 1st January, 2004 while the Prospect cohort was censored on January 1, 2007.

\section{Statistical analysis}

Cox proportional hazards analysis was used to examine the association between coffee and tea consumption and the incidence of breast cancer. Crude hazard ratios (HR) with 95\% confidence intervals were calculated for each coffee or tea intake category with the moderate consumers as reference. The HRs were further stratified into two BMI categories which have been reported as an effect modifier [19]. A cut-off point of $25 \mathrm{~kg} / \mathrm{m}^{2}$ was used to distinguish between normal and overweight women based on the WHO criteria. No separate analysis was carried out for premenopausal breast cancer subtypes as the number of premenopausal breast cancer cases was too small. Assessment of the estrogen receptor (ER) and progesterone receptor (PR) status was done in about 330 patients with breast cancer, and we analyzed the ER+/PR+ tumors separately. However, the numbers of $\mathrm{ER}+/ \mathrm{PR}-$ and $\mathrm{ER}-/ \mathrm{PR}-$ tumors were relatively too small to allow for further subgroup analysis.

Coffee and tea consumption was expected to be strongly associated with many lifestyle characteristics that could confound associations with breast cancer. In order to optimize confounder adjustment and avoid large Cox models, we first calculated propensity scores [31-33]. We used a logistic regression model to estimate the probability of drinking coffee for each participant as predicted by their age at recruitment, smoking status (never, past, current), educational status (low, intermediate, high), BMI, alcohol intake, energy intake, fat and fiber intake adjusted for energy (using nutrient residual model [34]), tea intake, physical activity level (inactive, moderately inactive, moderately active, active), ever prior use of oral contraceptives (yes, no), presence of hypercholesterolemia (yes, no), cohort (Prospect, Morgen), family history of breast cancer (yes, no), age at menarche, and parity. We also calculated the predicted probability of drinking tea for each participant using a similar logistic regression model which, instead, adjusted for coffee intake. The resulting propensity score (i.e., predicted probability) distributions for all the participants were divided into quintiles, and sufficient overlap of lifestyle characteristics by coffee and tea intake (yes/no) was evaluated as a prerequisite in propensity score modeling [31]. These propensity scores were considered as proxy for lifestyle of participants and subsequently added into the Cox regression model for adjustment of confounders.

The follow-up time was calculated from the date of enrollment of the study to the date of breast cancer diagnosis. Participants who, during follow-up, developed other type of cancers were censored on the date of diagnosis. Other participants were censored on loss to follow-up, at death or at the end of follow-up. The Cox proportional hazards assumption was examined by visually inspecting log-minus-log plots with no deviations detected.

Since using propensity scores alone would not help in identifying which covariates contributed the most as confounders in this study, we proceeded to identify such important covariates by dropping them one at a time and examining the changes in adjusted HRs as compared to the crude value. We did this for age, family history of breast cancer, age at menarche, parity, smoking, physical activity, education, and dietary exposures (combined intake of alcohol, total energy, saturated fat, fiber, and coffee for tea mutually).

Two-tailed $P$-values $<0.05$ and $95 \%$ confidence intervals for HR not including 1 were considered as statistically significant. All the statistical analyses were performed using SPSS for Windows version 16.0 (SPSS Inc., Chicago, USA).

\section{Results}

During an average of 9.6 years of follow-up, a total of 681 incidents of first invasive primary breast cancers were diagnosed among our study cohort of 27,323 women. Out of these, only 53 cases were pre-menopausal breast cancers (i.e., diagnosed before the age of 50 years), therefore, making it impossible for further meaningful analysis. The median age of this cohort at recruitment was 52.6 years with an age range of 20-70 years. Median coffee intake was 3.1 cups/day, and median tea intake was 2 cups/day. Overall, median BMI was $25.0 \mathrm{~kg} / \mathrm{m}^{2}$, and about $10 \%$ of women reported a positive history of breast cancer in a first degree relative. Baseline characteristics of participants according to coffee/tea consumption are shown in Table 1. Many risk factors for breast cancer, including lifestyle characteristics, were associated with coffee and tea intake.

Prior to adjustment, coffee intake increased the risk of breast cancer in consumers by more than twofold as compared to non-consumers (crude HR; 2.25, 95\% CI; 1.30-3.90). However, following adjustment, this association attenuated and was not significant (adjusted HR; 1.17, 95\% CI; 0.65-2.12). Table 2 shows the breast cancer risk across various categories of coffee intake where no significant association was observed in the overall or BMI 
Table 1 Relation of coffee and tea consumption with selected demographic, lifestyle, and breast cancer risk characteristics in 27,323 participants

\begin{tabular}{|c|c|c|c|c|c|c|}
\hline & 0 cup & $0.1-1.0$ cups & $1.1-2.0$ cups & $2.1-3.0$ cups & $3.1-5.0$ cups & $>5$ cups \\
\hline \multicolumn{7}{|l|}{ Daily coffee consumption } \\
\hline No. of participants & 1368 & 4667 & 3709 & 3896 & 7782 & 5901 \\
\hline Age (years) & 50.1 & 55.0 & 54.5 & 54.6 & 54.6 & 52.7 \\
\hline High educational level (\% [n]) & $21.8(297)$ & $18.7(870)$ & $23.3(863)$ & $22.1(860)$ & $16.6(1285)$ & $13.4(791)$ \\
\hline Tea intake (cups/day) & 5.0 & 3.0 & 3.0 & 2.0 & 2.0 & 1.0 \\
\hline Soft drink intake (cups/day) & 0.077 & 0.066 & 0.067 & 0.069 & 0.065 & 0.072 \\
\hline BMI $\left(\mathrm{kg} / \mathrm{m}^{2}\right)$ & 24.9 & 25.1 & 25.0 & 25.0 & 25.0 & 26.0 \\
\hline Low physical activity (\% [n]) & $10.4(142)$ & $9.8(458)$ & $8.4(310)$ & $7.7(299)$ & $6.9(534)$ & $9.0(529)$ \\
\hline Current smoker (\% [n]) & $17.8(244)$ & 20.9 (974) & $20.9(774)$ & $23.4(911)$ & $26.4(2052)$ & $44.4(2616)$ \\
\hline Alcohol intake (g/week) & 2.2 & 14.2 & 24.1 & 29.0 & 29.7 & 26.2 \\
\hline Total energy intake (kcal/day) & 1747 & 1730 & 1806 & 1818 & 1830 & 1857 \\
\hline Saturated fat intake $(\mathrm{g} / \text { day })^{\mathrm{a}}$ & 31.5 & 32.0 & 32.6 & 33.0 & 33.6 & 34.4 \\
\hline Fiber intake $(\mathrm{g} / \text { day })^{\mathrm{a}}$ & 22.1 & 23.6 & 23.6 & 23.5 & 23.6 & 23.6 \\
\hline Ever use of oral contraceptives $(\%[n])$ & $81.2(1109)$ & $72.2(3360)$ & $74.2(2746)$ & 72.9 (2838) & $70.6(5485)$ & $74.5(4388)$ \\
\hline Breast cancer in 1 st degree relative $(\%[n])$ & $7.1(96)$ & $9.9(458)$ & $9.5(350)$ & $10.2(393)$ & $11.3(871)$ & $10.1(588)$ \\
\hline Postmenopausal (\% [n]) & $33.8(462)$ & $64.5(3009)$ & $65.6(2433)$ & $67.4(2626)$ & $73.3(5708)$ & $67.0(3953)$ \\
\hline Ever use of postmenopausal hormone $(\%[\mathrm{n}])$ & $12.7(126)$ & $20.3(840)$ & $21.6(716)$ & $21.9(775)$ & $21.2(1531)$ & $20.0(1095)$ \\
\hline Age at menarche (years) & 13 & 13 & 13 & 13 & 13 & 13 \\
\hline Parity (number of children) & 2 & 2 & 2 & 2 & 2 & 2 \\
\hline \multicolumn{7}{|l|}{ Daily tea consumption } \\
\hline No. of participants & 2281 & 7462 & 5707 & 3336 & 5268 & 3269 \\
\hline Age (years) & 53.1 & 52.3 & 53.8 & 54.9 & 56.4 & 54.8 \\
\hline High educational level (\% [n]) & $7.4(168)$ & $14.8(1105)$ & $16.1(916)$ & $19.7(656)$ & $22.3(1171)$ & $29.1(950)$ \\
\hline Coffee intake (cups/day) & 4.5 & 3.6 & 3.6 & 2.7 & 2.7 & 1.5 \\
\hline Soft drinks intake (cups/day) & 0.077 & 0.092 & 0.077 & 0.060 & 0.053 & 0.040 \\
\hline BMI $\left(\mathrm{kg} / \mathrm{m}^{2}\right)$ & 26.0 & 25.5 & 25.1 & 25.0 & 25.0 & 24.9 \\
\hline Low physical activity (\% [n]) & $13.6(311)$ & $9.3(692)$ & $8.1(461)$ & $7.4(248)$ & $6.1(322)$ & $7.3(238)$ \\
\hline Current smoker (\% [n]) & $50.3(1144)$ & $36.8(2742)$ & $26.7(1524)$ & $20.6(687)$ & $16.9(889)$ & $17.9(585)$ \\
\hline Alcohol intake (g/week) & 17.0 & 24.2 & 23.9 & 24.4 & 23.9 & 21.9 \\
\hline Total energy intake (kcal/day) & 1751 & 1827 & 1813 & 1813 & 1828 & 1785 \\
\hline Saturated fat intake (g/day) $)^{\mathrm{a}}$ & 34.1 & 33.7 & 33.2 & 33.1 & 32.9 & 32.5 \\
\hline Fiber intake $(\mathrm{g} / \text { day })^{\mathrm{a}}$ & 22.8 & 22.8 & 23.3 & 23.9 & 24.3 & 24.6 \\
\hline Ever use of oral contraceptives (\% [n]) & $69.2(1577)$ & $77.6(5777)$ & $73.2(4175)$ & $71.4(2375)$ & $69.2(3640)$ & $72.9(2382)$ \\
\hline Breast cancer in 1 st degree relative $(\%[n])$ & $10.1(228)$ & $9.5(699)$ & $10.9(618)$ & $9.8(324)$ & $10.6(553)$ & $10.3(334)$ \\
\hline Post menopausal (\% [n]) & $67.3(1536)$ & $58.7(4381)$ & $67.6(3856)$ & $69.4(2316)$ & $74.2(3907)$ & $67.1(2195)$ \\
\hline Ever use of post menopausal hormones $(\%[\mathrm{n}])$ & $18.1(384)$ & $20.1(1323)$ & $21.7(1128)$ & $20.9(634)$ & $20.5(994)$ & $21.3(620)$ \\
\hline Age at menarche (years) & 13 & 13 & 13 & 13 & 13 & 13 \\
\hline Parity (number of children) & 2 & 2 & 2 & 2 & 2 & 2 \\
\hline
\end{tabular}

All continuous variables are expressed as median, while the categorical variables are expressed as percentages

${ }^{a}$ Energy-adjusted values

specific groups. Restriction of the analysis to only postmenopausal breast cancer cases (i.e., diagnosed after the age of 50 years) also resulted in similar results, despite additionally adjusting for use of postmenopausal hormones (results not shown).

Tea intake was associated with risk of breast cancer neither in the crude analysis nor in the adjusted analysis
(Table 3). The results did not change with either stratification to BMI status, or by restriction of the analysis to postmenopausal women.

When coffee and tea intakes were combined, the Cox analysis across the different categories did not show any significant association with the risk of breast cancer (results not shown). 
Table 2 Coffee consumption and risk of breast cancer stratified by BMI status

\begin{tabular}{|c|c|c|c|c|c|c|c|}
\hline Daily coffee intake & $n$ & 0 cup & $0.1-1.0$ cups & $1.1-2.0$ cups & 2.1-3.0 cups & 3.1-5.0 cups & $>5$ cups \\
\hline No. of participants & 27,323 & 1368 & 4667 & 3709 & 3896 & 7782 & 5901 \\
\hline \multicolumn{8}{|l|}{ Total breast cancers } \\
\hline No. of cases & 681 & 13 & 110 & 108 & 100 & 198 & 152 \\
\hline Crude HR & & 0.47 & 1.00 & 1.23 & 1.00 & 1.07 & 1.06 \\
\hline$(95 \% \mathrm{CI})$ & & $(0.27-0.84)$ & & $(0.94-1.62)$ & $(0.75-1.32)$ & $(0.84-1.35)$ & $(0.83-1.37)$ \\
\hline Adjusted HR & & 0.86 & 1.00 & 1.20 & 0.93 & 0.97 & 0.94 \\
\hline$(95 \% \mathrm{CI})^{\mathrm{a}}$ & & $(0.47-1.59)$ & & $(0.90-1.60)$ & $(0.69-1.25)$ & $(0.75-1.25)$ & $(0.72-1.24)$ \\
\hline \multicolumn{8}{|l|}{ Women with $B M I \leq 25 \mathrm{~kg} / \mathrm{m}^{2}$} \\
\hline No. of breast cancer cases & 313 & 8 & 44 & 52 & 52 & 95 & 62 \\
\hline Adjusted HR & & 1.22 & 1.00 & 1.37 & 1.11 & 1.20 & 1.09 \\
\hline$(95 \% \mathrm{CI})^{\mathrm{a}}$ & & $(0.53-2.80)$ & & $(0.88-2.13)$ & $(0.71-1.75)$ & $(0.81-1.78)$ & $(0.71-1.68)$ \\
\hline \multicolumn{8}{|l|}{ Women with BMI $>25 \mathrm{~kg} / \mathrm{m}^{2}$} \\
\hline No. of breast cancer cases & 368 & 5 & 66 & 56 & 48 & 103 & 90 \\
\hline Adjusted HR & & 0.62 & 1.00 & 1.12 & 0.83 & 0.83 & 0.84 \\
\hline$(95 \% \mathrm{CI})^{\mathrm{a}}$ & & $(0.24-1.57)$ & & $(0.76-1.65)$ & $(0.55-1.24)$ & $(0.59-1.16)$ & $(0.59-1.20)$ \\
\hline
\end{tabular}

$H R$ hazard ratio, $C I$ confidence interval

a Adjusted to propensity score (based on age, smoking status, educational status, BMI, alcohol intake, energy intake, energy-adjusted saturated fat intake, energy-adjusted fiber intake, tea intake, physical activity level, ever prior use of oral contraceptives, presence of hypercholesterolemia, family history of breast cancer, age at menarche, parity, and cohort)

Table 3 Tea consumption and risk of breast cancer stratified by BMI status

\begin{tabular}{|c|c|c|c|c|c|c|c|}
\hline Daily tea intake & & 0 cup & $0.1-1.0$ cups & $1.1-2.0$ cups & 2.1-3.0 cups & $3.1-5.0$ cups & $>5$ cups \\
\hline No. of participants & 27,323 & 2281 & 7462 & 5707 & 3336 & 5268 & 3269 \\
\hline \multicolumn{8}{|l|}{ All women } \\
\hline No. of breast cancer cases & 681 & 53 & 195 & 146 & 83 & 128 & 76 \\
\hline Crude HR & & 0.84 & 1.00 & 0.97 & 0.91 & 0.87 & 0.86 \\
\hline$(95 \% \mathrm{CI})$ & & $(0.61-1.15)$ & & $(0.78-1.21)$ & $(0.70-1.18)$ & $(0.69-1.10)$ & $(0.65-1.13)$ \\
\hline Adjusted HR & & 0.74 & 1.00 & 0.93 & 0.90 & 0.83 & 0.83 \\
\hline$(95 \% \mathrm{CI})^{\mathrm{a}}$ & & $(0.52-1.05)$ & & $(0.74-1.17)$ & $(0.68-1.19)$ & $(0.65-1.06)$ & $(0.62-1.11)$ \\
\hline \multicolumn{8}{|l|}{ Women with $B M I \leq 25 \mathrm{~kg} / \mathrm{m}^{2}$} \\
\hline No. of breast cancer cases & 313 & 21 & 93 & 53 & 41 & 67 & 38 \\
\hline Adjusted HR & & 0.63 & 1.00 & 0.72 & 0.80 & 0.86 & 0.68 \\
\hline$(95 \% \mathrm{CI})^{\mathrm{a}}$ & & $(0.36-1.10)$ & & $(0.51-1.03)$ & $(0.53-1.20)$ & $(0.60-1.21)$ & $(0.45-1.05)$ \\
\hline \multicolumn{8}{|l|}{ Women with BMI $>25 \mathrm{~kg} / \mathrm{m}^{2}$} \\
\hline No. of breast cancer cases & 368 & 32 & 102 & 93 & 42 & 61 & 38 \\
\hline Adjusted HR & & 0.85 & 1.00 & 1.13 & 1.01 & 0.80 & 1.03 \\
\hline$(95 \% \mathrm{CI})^{\mathrm{a}}$ & & $(0.54-1.33)$ & & $(0.84-1.54)$ & $(0.69-1.48)$ & $(0.56-1.13)$ & $(0.69-1.54)$ \\
\hline
\end{tabular}

$H R$ hazard ratio, $C I$ confidence interval

a Adjusted to propensity score (based on age, smoking status, educational status, BMI, alcohol intake, energy intake, energy adjusted saturated fat intake, energy adjusted fiber intake, coffee intake, physical activity level, ever use of oral contraceptives, presence of hypercholesterolemia, family history of breast cancer, age at menarche, parity, and cohort)

The ER/PR status was assessed in about $48 \%$ of breast cancer patients. Among them, 192 were $\mathrm{ER}+/ \mathrm{PR}+, 51$ were $\mathrm{ER}+/ \mathrm{PR}-$, and 45 were $\mathrm{ER}-/ \mathrm{PR}-$. Overall, there was no association between coffee or tea intake and risk of breast cancer among the patients with $\mathrm{ER}+/ \mathrm{PR}+$ tumor. Adjusted HR for coffee consumers versus non-consumers was $1.18,95 \% \mathrm{CI}$; 0.37-3.78, while adjusted HR for tea consumers versus non-consumers was $1.35,95 \%$ CI; $0.77-$ 2.39 .

Since there was a significant attenuation in risk of breast cancer in relation to coffee consumption following adjustment with the propensity scores, we proceeded to 
identify which covariate contributed to this effect. We found that dietary exposures in combination contributed most to the change in breast cancer risk among coffee consumers, followed by age, age at menarche, and parity. Family history of breast cancer, smoking, alcohol, and physical activity did not contribute to the change in risk among the coffee drinkers.

In order to avoid spurious reverse associations, we restricted our analyses to breast cancer cases occurring after 2 years of follow up. It did not materially change the observed HRs (not shown).

\section{Discussion}

We found no statistically significant association between coffee or tea consumption and risk of breast cancer. These results were similar for lean and overweight women as well as when restricting analysis to postmenopausal breast cancers.

The strength of our study is its prospective nature, the relatively large sample size involving 27,323 women, and solid confounder adjustment which accounted for the risk factors for breast cancer and various lifestyle-related variables, summarized to a single propensity score and subsequently adjusted for. Even though most of the previous prospective studies adjusted for multiple confounders mainly age, risk factors of breast cancer, smoking (except in 1 study [16]), and total energy intake, many did not adjust for other important nutritional variables such as fiber intake (except two studies $[13,16]$ ), saturated fat intake, alcohol intake (except 4 studies [13-16]), or mutually adjusted coffee for tea intake since these beverages are inversely correlated (except two studies [13, 14]). Even if these variables are solely not capable of changing the hazard ratios for developing breast cancer drastically, they may in combination confound the association between coffee or tea intake and risk of breast cancer.

Since the measurement of coffee and tea intake was done at baseline only, we are uncertain about the effects of participants subsequently changing their pattern of coffee/tea consumption. However, this would only pose a problem if change in coffee or tea consumption occurred selectively in a particular group of participants (i.e., those subsequently developing breast cancer or vice versa), but this is highly unlikely. Anyhow, we attempted to deal with this problem by excluding those with prevalent cancer at baseline, and censoring participants with cancer at the time of their diagnosis, which may have induced change in their dietary habits.

Even though we did not have information on the type of tea consumed by our study population, we are aware that the percentage of green tea consumption is very low in the Western population, where black tea consumption is dominant. Since there was no information on history of prior benign breast disease, we were unable to incorporate this risk factor of breast cancer into our present analysis. We also did not have genetic information, e.g., BRCA1 or BRCA2 mutations.

Findings of previous epidemiological studies on the association of coffee consumption with breast cancer risk, however, have been inconsistent and even contradictory, possibly due to issues of study design and methodology. Subsequent to the WCRF report in 2007, two large prospective cohort studies revealed that there is no association between the consumption of these beverages and risk of breast cancer $[15,16]$. Our study is, therefore, valuable since it further adds concrete and valid evidence to this finding. However, a recent meta-analysis of 18 studies showed a weak inverse association between coffee and tea intake and risk of breast cancer, where the pooled RR for the highest versus lowest coffee consumption level was $0.95(0.90-1.00)$ [35]. Anyhow, it is possible that misclassification of coffee consumption may have occurred in this analysis due to variation in measurement of coffee or tea intake among studies which probably diluted the observed effects toward the null value.

Even though animal and cellular studies seem to show either a protective [23-25] or harmful [21, 22] effect of coffee and tea in relation to breast cancer, we were not able to demonstrate this in humans most probably due to higher doses of coffee/tea constituents used in laboratory studies or due to differences in causal factors of cancer in humans and animals [36]. It may also be possible that the counter effect among various constituents in these beverages leaves no net effect in humans.

From this study, it seems that the association between coffee and tea intake and risk of breast cancer is actually strongly confounded by lifestyle, especially with coffee where a significant crude HR of 2.25 (95\% CI, 1.30-3.90) comparing consumers versus non-consumers was substantially reduced to $1.17(0.65-2.12)$ after adjustment for lifestyle factors. In fact, this effect of adjustment indicates that the coffee (or tea) drinking-associated lifestyle seems important in respect to breast cancer. It is very likely that the various lifestyle determinants have acted synergistically in the causal pathway of breast cancer as suggested from the effect brought about by adjustment with the propensity score.

As for premenopausal breast cancers, we could not draw any conclusion since the number of cases was too small to perform a meaningful analysis. Therefore, we would need a bigger sample to capture any associations between coffee or tea intake and the risk of breast cancer among premenopausal women.

In conclusion, our findings support the view that coffee and tea consumption is not related to the risk of breast cancer in women. 


\section{References}

1. Parkin MD, Bray F, Ferlay J, Pisani P (2005) Global Cancer Statistics, 2002. CA Cancer J Clin 55:74-108

2. World Cancer Research Fund/American Institute for Cancer Research (2007) Food, nutrition, physical activity, and the prevention of cancer: a global perspective. Washington DC: AICR

3. McLaughlin CC, Mahoney MC, Nasca PC et al (1992) Breast cancer and methylxanthine consumption. Cancer Causes Control 3(2): $175-178$

4. Ewertz M, Gill C (1990) Dietary factors and breast cancer risk in Denmark. Int J Cancer 46(5):779-784

5. Tavani A, Pregnolato A, La Vecchia C et al (1998) Coffee consumption and the risk of breast cancer. Eur J Cancer Prev $7(1): 77-82$

6. Wu AH, Yu MC, Tseng CC et al (2003) Green tea and risk of breast cancer in Asian Americans. Int J Cancer 106:574-579

7. Mannisto S, Pietinen P, Virtanen V et al (1999) Diet and the risk of breast cancer in a case-control study: does the threat of disease have an influence on recall bias? J Clin Epidemiol 52:429-439

8. Kumar N, Titus-Ernstoff L, Newcomb PA et al. (2009) Tea consumption and risk of breast cancer. Cancer Epidemiol Biomarkers Prev 18(1):341-345

9. Baker JA, Beehler GP, Sawant AC et al (2006) Consumption of coffee, but not black tea, is associated with decreased risk of premenopausal breast cancer. J Nutr 136:166-171

10. Zhang M, Holman CDJ, Huang JP, Xie X (2007) Green tea and the prevention of breast cancer: a case control study in Southeast China. Carcinogenesis 28(5):1074-1078

11. Rosenberg L, Miller DR, Helmrich SP et al (1985) Breast cancer and the consumption of coffee. Am J Epidemiol 122(3):391-399

12. Zheng W, Doyle TJ, Kushi LH et al (1996) Tea consumption and cancer incidence in a prospective cohort study of postmenopausal women. Am J Epidemiol 144:175-182

13. Folsom AR, McKenzie DR, Bisgard KM et al (1993) No association between caffeine intake and postmenopausal breast cancer incidence in the Iowa Women's Health Study. Am J Epidemiol 138:380-383

14. Suzuki Y, Tsubono Y, Nakaya N et al (2004) Green tea and the risk of breast cancer: pooled analysis of two prospective studies in Japan. Br J Cancer 90:1361-1363

15. Ganmaa D, Willet WC, Li TY et al (2008) Coffee, tea, caffeine and risk of breast cancer: a 22-year follow-up. Int J Cancer 122: 2071-2076

16. Ishitani K, Lin J, Manson JE (2008) Caffeine consumption and the risk of breast cancer in a large prospective cohort of women. Arch Intern Med 168(18):2022-2031

17. Michels KB, Holmberg L, Bergkvist L et al (2002) Coffee, tea, and caffeine consumption and breast cancer incidence in a cohort of Swedish Women. Ann Epidemiol 12(1):21-26

18. Hirvonen T, Mennen LI, de Bree A et al (2006) Consumption of antioxidant-rich beverages and risk for breast cancer in French women. Ann Epidemiol 16:503-508

19. Vatten LJ, Solvoll K, Loken EB (1990) Coffee consumption and the risk of breast cancer. A prospective study of 14, 593 Norwegian women. Br J Cancer 62(2):267-270
20. Scalbert A, Williamson G (2000) Dietary intake and bioavailability of polyphenols. J Nutr 130:2073-2085

21. Welsch CW, Scieszka KM, Senn ER et al (1983) Caffeine (1, 3, 7-trimethylxanthine), a temperate promoter of DMBA-induced rat mammary gland carcinogenesis. Int J Cancer 32(4):479-484

22. Trosko JE, Chu HY (1973) Inhibition of repair of UV-damaged DNA by caffeine and mutation induction in Chinese hamster cells. Chem Biol Interact 6(5):317-322

23. Wang LQ (2002) Mammalian phytoestrogens: enterodiol and enterolactone. J Chromatogr B Analyt Technol Biomed Life Sci 777(1-2):289-309

24. Adlercreutz H (2002) Phyto-oestrogens and cancer. Lancet Oncol 3:364-373

25. Brooks JD, Thompson LU (2005) Mammalian lignans and genistein decrease the activities of aromatase and 17beta-hydroxysteroid dehydrogenase in MCF-7 cells. J Steroid Biochem Mol Biol 94(5):461-467

26. Riboli E, Kaaks R (1997) The EPIC project: rationale and study design. Int J Epidemiol 26:S6-S14

27. Boker LK, Van Noord PAH, Van Der Schouw YT et al (2001) Prospect-EPIC Utrecht: study design and characteristics of the cohort population. Eur J Epidemiol 17:1047-1053

28. Blokstra A, Seidell JC, Smit HA et al. (1998) The monitoring project on risk factors for chronic diseases (MORGEN project) annual report 1997 (in Dutch). RIVM (National Institute for Public Health and the Environment), Bilthoven, Netherlands

29. Ocké MC, Bueno-de-Mesquita HB, Goddijn HE et al (1997) The Dutch EPIC Food Frequency Questionnaire I. Description of the questionnaire, and relative validity and reproducibility for food groups. Int J Epidemiol 26:S37-S48

30. Ocké MC, Bueno-de-Mesquita HB, Pols MA, Smit HA, Van Staveren WA, Kromhout D (1997) The Dutch EPIC Food Frequency Questionnaire II. Relative validity and reproducibility for nutrients. Int J Epidemiol 26:S49-S58

31. Fitzmaurice $\mathrm{G}$ (2006) Confounding: propensity score adjustment. Nutrition 22:1214-1216

32. D'Agostino RB (1998) Propensity score methods for bias reduction in the comparison of a treatment to a non-randomized control group. Stat Med 17:2265-2281

33. Kurth T, Walker AM, Glynn RJ et al (2006) Results of multivariable logistic regression, propensity matching, and propensitybased weighting under conditions of nonuniform effect. Am J Epidemiol 163:262-270

34. Willett WC, Howe GR, Kushi LH (1997) Adjustment for total energy intake in epidemiologic studies. Am J Clin Nutr 65: 1220S-1228S

35. Tang N, Zhou B, Wang B, et al. (2009) Coffee consumption and risk of breast cancer: a metaanalysis. Am J Obstet Gynecol 200(3):290.e1-290.e9

36. Luo J, Inoue M, Iwasaki M et al (2007) Green tea and coffee intake and risk of pancreatic cancer in a large scale, population based cohort study in Japan (JPHC study). Eur J Cancer Prev 16(6):542-548 